\title{
PLANIFICACIÓN DEL DESARROLLO A LARGO PLAZO BAJO LA TEORÍA DEL DESARROLLO PROFESIONAL EN TAIWÁN
}

\author{
Joey TSENG \\ Universidad Nacional de Sun Yat-sen. (Taiwán) \\ Jorge Martín Martín \\ Universidad Autónoma de Madrid (España) \\ j.mart90@hotmail.com \\ Fecha de recepción: Noviembre 2018 \\ Fecha de aceptación: Enero 2019
}

http://doi.org/10.15366/citius2019.12.1.004

\section{Resumen:}

Los atletas reciben innumerables aplausos en su vida deportiva, pero su verdadera prueba de vida viene con su retirada como deportista (Kerr y Dacyshyn, 2000; Lally, 2007). Una etapa obligatoria para todos los atletas es la transferencia de su vida deportiva a una vida laboral. En la actualidad el mercado laboral y nuestras vidas han cambiado, debido a la influencia de la tecnología y el desarrollo de la ciencia. La gestión de la vida deportiva se ha convertido en un nuevo reto, a la vez que una oportunidad para los atletas de desarrollar sus capacidades y oficios. Cada vez más y más atletas rechazan los oficios tradicionales como entrenadores y profesores. En su lugar eligen formarse en negocios, medios de comunicación, tecnología e incluso crear su propio negocio. Los buenos entrenadores permiten a sus pupilos crecer personalmente, dándoles a ellos una misión superior, convertirse en referentes sociales.

En el espectro de Recursos Humanos, los atletas son un grupo especial, debido a que tras haber tenido una buena vida atlética; a corto plazo, deben de vivir una transición inevitable (Huang, 2002). Los sistemas de gobierno y el pensamiento tradicional de las personas de Taiwán hacen pensar al atleta que solo puede elegir un camino: el deportivo o el académico. Este trabajo se ha centrado en la política que ofrece el estado a los atletas taiwaneses, con el fin de preparar y planificar su inserción laboral futura. Al mismo tiempo, los deportistas deben romper con el marco tradicional y formarse académica y personalmente.

Palabras Clave: Teoría del desarrollo profesional, Atletas Taiwán, Planificación del desarrollo a largo plazo.

Title: THE LONG-TERM CAREER PLANNING OF ATHLETES UNDER THE THEORY OF CAREER DEVELOPMENT IN TAIWAN CASES

\begin{abstract}
:
Athletes receive countless applause in the competitive arena, but the life test just begins after retirement (Kerr \& Dacyshyn, 2000; Lally, 2007). Career track transfer is a compulsory course for every athlete. In digital age, development of science and technology already change our daily life, and the labour market has also changed. Diversified career management has become a new challenge and opportunity for the development of athletes' career and capacity. More and more athletes escape from traditional career choices such as coaches and teachers, and instead to devote themselves to business, media, technology and other industries, including starting a business. Good job transfer coaches enable athletes to re-recognize themselves and pursue self-growth, giving athletes a higher mission, thus becoming a benchmark and model for society.
\end{abstract}

In the spectrum of human resources, athletes are a most special group, and the wonderful but short-term athletic career makes transition inevitable (Huang, 2002). In Taiwan traditional thinking and 
government systems, sports training or academics can only choose one, which is the biggest obstacle to the entry of sports talents into the long-term sports career period. This study will focus on Taiwanese athletes' long-term career planning and policy content in practice oriented, emphasizing the diversification of athletes' skill in the workplace in order to prepare for their future work environment. At the same time, athletes should break away from traditional framework by cultivating second expertise and self-improvement.

Key words: Theory of career development, Athletes Taiwan, Long-term career planning

\section{1. «¿Qué es lo siguiente que debe hacer el deportista?»}

Los deportistas se preguntan de vez en cuando, qué será de ellos cuando se terminen sus trayectorias deportivas. Esto es debido a que la edad media de retirada de un deportista de alto nivel son los 30 años edad. Por tanto, el deportista debe pensar qué quiere hacer después de que se termine su vida deportiva, dado que, tras ésta, deberá de elegir un segundo trabajo. Algunos de los problemas más comunes que influyen en el desarrollo laboral de los atletas son: a nivel psicológico — derivados de tener que encontrar trabajo - a nivel de formación — déficit- y a nivel de experiencia — falta de hábitos en su nuevo oficio.

\section{La propuesta y la connotación de la teoría del desarrollo profesional.}

En la década de 1950 se creó la teoría de desarrollo profesional, basada en: la psicología diferencial, el autoconcepto, la psicología del desarrollo y la investigación sobre el desarrollo del comportamiento profesional. El desarrollo de la carrera profesional tiene tres niveles.

En primer lugar, el desarrollo de lo profesional. En base a la edad, el desarrollo de la profesionalidad se divide en cinco etapas: Crecimiento (desde el nacimiento hasta los 14 años), exploración (de los 15 a los 24 años), establecimiento (entre los 25 a los 44 años), mantenimiento (de los 45 a los 64 años) y declive (de los 65 años hasta la muerte). Cada etapa del desarrollo de la carrera profesional tiene diferentes funciones y características. La finalización de cada etapa tratada del desarrollo de la carrera profesional afecta directamente al desarrollo de las siguientes etapas del desarrollo.

En segundo lugar, está la amplitud del desarrollo de la carrera profesional. Este nivel trata sobre los nueve diferentes roles que tiene una persona en su vida: niño, estudiante, ocio, ciudadano, trabajador, pareja, padre o madre y jubilado. Con la edad estos roles irán apareciendo uno tras otro en las diferentes etapas, incluso interaccionarán entre ellos en algunas situaciones.

En tercer lugar, está la profundidad del desarrollo de una carrera profesional. En este nivel se desarrollan los juegos de roles en los individuos.

El desarrollo de una carrera profesional es un proceso irreversible de una dinámica continua. El pasado forma parte del presente y al mismo tiempo afecta al futuro. El comportamiento actual y las expectativas de futuro de una persona tendrán un impacto en el desarrollo de su carrera profesional. La planificación y orientación profesional son imprescindibles para poder ayudar a promover y progresar en el desarrollo de una carrera profesional. Los beneficios de tener un plan de desarrollo profesional correcto son:

- Identificar la etapa de desarrollo profesional en la que se encuentra y fijar unas metas a alcanzar en cada etapa.

- Ayudar al estudiante a aclarar su autoconcepto, ya que si mejora su autoconcepto aumentará su madurez vocacional. Por eso, se hace necesaria una ayuda que contribuya a relacionar su conocimiento con información ocupacional. 
- Exponer a los estudiantes una amplia gama de profesiones, para ir acotándolas con el paso del tiempo. Se debe de tener en cuenta el estilo de vida y las asignaturas cursadas por el individuo, con el fin de que éste encuentre su vocación.

- La experiencia laboral es vital. Se pueden usar los roles para crear situaciones de trabajo real. La teoría de desarrollo de carrera profesional permite realizar cambios en uno mismo. Estos cambios son muy apropiados para aplicarlos en un trabajo del siglo XXI.

\section{El concepto de las etapas de vida y sus funciones en los atletas de Taiwán}

\begin{tabular}{|c|c|}
\hline $\begin{array}{l}\text { Comienza con el } \\
\text { nacimiento y } \\
\text { termina alrededor } \\
\text { de los } 14 \text { años. }\end{array}$ & $\begin{array}{l}\text { Características } \\
\text { Los atletas jóvenes, a través de su capacidad atlética y sus intereses } \\
\text { personales, eligen lo que aman, ya bien sea de forma activa o pasiva } \\
\text { Sub-etapas } \\
\text { La formación especializada se lleva a cabo desde la base de la escuela } \\
\text { hasta el instituto. } \\
\text { El concepto de confianza en uno mismo se va estableciendo poco a } \\
\text { poco durante este periodo. } \\
\text { Funciones o Cometidos } \\
\text { Desarrollar qué tipo de persona quiere ser. } \\
\text { Descubrir y desarrollar los talentos atléticos y habilidades que tenga el } \\
\text { individuo. }\end{array}$ \\
\hline $\begin{array}{l}\text { Entre los } 15 \text { y } 24 \\
\text { años }\end{array}$ & $\begin{array}{l}\text { Características } \\
\text { Entre los } 15 \text { y } 24 \text { años es una etapa importante en la vida de un atleta. } \\
\text { El papel desempeñado por los atletas en este período es principalmente } \\
\text { entrenar y estudiar. No hay ninguna otra función o tarea a la que } \\
\text { dedicar tiempo y energía más que la de desarrollar su formación en los } \\
\text { deportes, a la vez que se va desarrollando. } \\
\text { A largo plazo, la formación profesional sistemática y disciplinada, } \\
\text { ayuda al individuo a crear un autoaprendizaje, a realizar exámenes de } \\
\text { conciencia. El objetivo es orientar al individuo en la elección de una } \\
\text { profesión acorde para él. } \\
\text { Sub-etapas } \\
\text { Entre los } 20 \text { y } 24 \text { años, algunos excelentes atletas emergen en deportes } \\
\text { importantes. } \\
\text { La finalización de su etapa de exploración afectará directamente a la } \\
\text { elección profesional, confirmando su desarrollo laboral a largo plazo y } \\
\text { su toma de decisiones. } \\
\text { Funciones o Contenidos } \\
\text { Guía a sus atletas para establecer un sentido de profesionalismo y } \\
\text { establece los objetivos de desarrollo claros para el desarrollo } \\
\text { profesional. } \\
\text { Un marco de meta clara alrededor de la misión de objetivo. } \\
\text { Prepararse para el salto al profesionalismo. }\end{array}$ \\
\hline
\end{tabular}




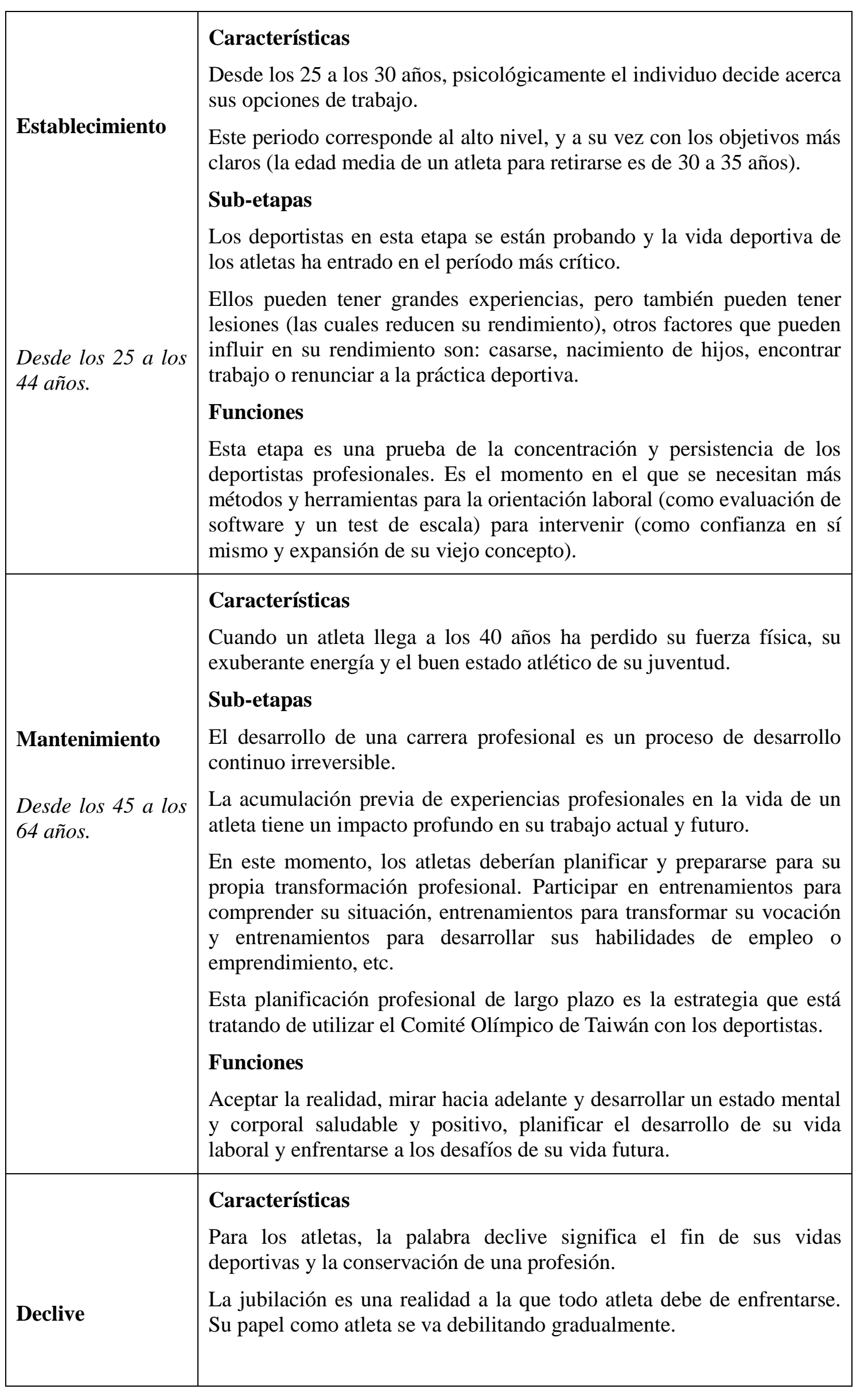


Desde los 65 años hasta la muerte
Después de su retirada, ellos entran en un nuevo campo profesional y se enfrentan a un espacio de vida más amplio, lo que dará a los atletas retirados más roles. Estos roles están estrechamente relacionados y pueden afectar a su desarrollo profesional futuro.

La particularidad de la ocupación de un atleta y la naturaleza limitada de la vida deportiva hacen que esta etapa sea mucho más temprana que en otras ocupaciones.

\section{Sub-etapas}

Esta etapa incluye información sobre el empleo e introducción de políticas, asesoramiento empresarial, mejora de la capacidad en el lugar de trabajo, pasantías de empleo y referencias profesionales.

Esta etapa incluye información sobre el empleo e introducción de políticas, asesoramiento empresarial, mejora de la capacidad en el lugar de trabajo, prácticas y referencias profesionales.

\section{Funciones}

Cómo lograr con éxito la transformación profesional, mejor darse cuenta de su propio valor y el desarrollo profesional después de la jubilación, es la principal tarea de los atletas en esta etapa.

La teoría del desarrollo de la carrera profesional muestra una buena aplicación para resolver el dilema del empleo de los atletas y la confusión de desarrollo profesional. Porque los atletas están en un ambiente de formación relativamente cerrado durante mucho tiempo, los deportistas no son conscientes de la sociedad, el nivel de cultura es bajo y las posibilidades de encontrar un empleo en el que se carezca de habilidades motoras son reducidas. Por tanto, los atletas se jubilan y encontrar un empleo es difícil para ellos. La carrera de desarrollo profesional intenta resolver los problemas de empleo con los atletas retirados. Entonces, basándose en la experiencia existente, es de gran importancia usar la teoría del desarrollo de una carrera profesional para guiar a los atletas en sus futuras vidas laborales.

\section{El desarrollo de la orientación de los atletas es una tendencia inevitable en el futuro}

Tras la retirada los atletas, deben lograr un desarrollo en su carrera profesional y este modelo se ha convertido en un modelo para transformar su empleo. En Taiwán, el departamento de deportes del ministerio de educación y el comité olímpico de China Taipéi, promueven conjuntamente un programa para facilitar la transformación de su vida deportiva a una vida laboral, tras la retirada del atleta de su deporte, para ello comienzan a planificar su inserción al mundo laboral lo antes posible. Por ejemplo, Chen Yi-An (陳怡安) en un principio no consideró conveniente esta técnica de desarrollo, pero luego ella se convirtió en una taekwondista. En el año 1988 acudió a los Juegos Olímpicos de Seúl, Allí es donde ella comenzó su camino hacia la primera medalla de oro olímpica en la historia de Taiwán. Cuando ella se retiró, ella entró en un mercado laboral altamente competitivo. Buscando trabajo de reportera de deportes, periodista y presentadora. Pero, lo que a ella le gustaba era la artesanía, así pues, empezó un negocio de venta de jabón fabricado a mano, junto con amigos y amigas. Ahora Chen Yi-An se ha convertido en una de las fragancias de jabón que más se venden en los supermercados. Weng Ming-Yi(翁明), es el único atleta que ha representado a Taiwán en tres Juegos Olímpicos de invierno. Desde que se convirtió en un instructor de esquí en 1985 en Europa, él volvió a Taiwán para iniciar un negocio. Él ha creado una nueva tendencia de desplazamiento que es esquiar sobre un tipo de hierba deslizante creada por el mismo. Ahora es el fundador de la famosa hierva-deslizante marca Taiwán y se llama «Grass-flying» (HierbaVuelo). Después de retirarse, se convirtió en el jefe de tres tiendas de esquí. Además, él es el actual vicepresidente de la asociación de esquí de Taiwán. 
En esta etapa de la jubilación, muchos atletas profesionales se transforman en múltiplos roles: jefes, entrenadores, maridos, esposas, padres, madres, hijos, hijas, etc. Sólo planificando la carrera profesional de los deportistas se consigue que ellos transformen su vida laboral. La acumulación de trabajo y los logros en su deporte unido a su valiosa experiencia atlética, a su voluntad y a su espíritu de sacrificio. Por supuesto, esto no se podría llevar a cabo sin una política de deportes nacional y la planificación de carrera profesional de los atletas. Lin PengSoong (2003), quien durante mucho tiempo ha observado y analizado a los deportistas Olímpicos de Taiwán, ha propuesto que los atletas olímpicos que se interesen por su vida futura y que esta tenga relación con su pasado deportivo.

Según Lin (2003), es muy prometedor centrarse en el trabajo y oportunidades que te da tu experiencia deportiva en unos Juegos Olímpicos. Con el advenimiento de la era digital, más las nuevas formas de trabajo empresarial, los atletas deben aprender a planear su futuro de ahora en adelante.

\section{La orientación laboral de los atletas de Taiwán bajo la teoría del desarrollo de su carrera profesional.}

En Taiwán, la introducción de la teoría de desarrollo de carreras profesionales es un objetivo y una condición necesaria para lograr el crecimiento general de los atletas y las conexiones con el mundo laboral. En la actualidad, el modo de trabajar esta técnica con atletas taiwaneses es la formación por medio de cursos a cambio de sus logros deportivos. Por lo tanto, es difícil asegurar la continuidad del aprendizaje y el aprendizaje profundo en el futuro; el antiguo concepto de deportes sólo se preocupaba por los atletas. En esta etapa, con la teoría de desarrollo de carrera y la introducción en los atletas para el análisis ayudará a los atletas a entender y promover el más amplio crecimiento y desarrollo de la vida profesional.

Para completar la planificación de su vida laboral los atletas, deben de comprender el concepto de planificación de su vida laboral futura, además este concepto también debe estar arraigado profundamente en los corazones de la sociedad. Este estudio describe los cinco pasos que se dan en Taiwán y que guían a los atletas a completar la planificación.

Desde la correcta planificación a largo plazo los atletas conseguirán desarrollar sus carreras profesionales de una forma: científica, sistemática y continua. Con el fin de garantizar el mejor desarrollo posible de sus carreras profesionales por medio de la estabilidad y la prosperidad de toda la sociedad. Si realmente se explora las raíces del desarrollo profesional, la solución principal está en las ideas y métodos. Por un lado, tenemos que cambiar nuestra mentalidad. Ya sean gerentes, entrenadores y líderes de equipos o atletas individuales, debemos establecer el concepto de desarrollo de carrera profesional a tiempo completo. Las orientaciones profesionales deben ejecutarse a través de todas las etapas de desarrollo de la carrera profesional de los atletas. Los atletas deben establecerse una planificación. Ejercer la iniciativa subjetiva en la planificación de la carrera; por otro lado, a través de la planificación de carrera profesional efectiva, desarrollo de herramientas de evaluación, construcción de plataformas de trabajo, establecimiento de sistemas de apoyo. Debe de integrar al gobierno, sociedad, familias, individuos, con el fin de establecer un sistema de apoyo del trabajo de orientación integral para deportistas, sociedad, familias e individuos y prestar apoyo para la orientación profesional de los atletas de Taiwán, con el fin de garantizar el mejor desarrollo de los atletas en sus vidas. La información de esta investigación puede ser utilizada como referencia por las autoridades de Taiwán. 
Crecimiento:

Tener los conceptos de auto planificación a largo plazo.
El concepto de planificación de carrera está profundamente arraigado en las mentes de las personas, integrado en el entrenamiento diario, la competencia, la educación escolar y la vida familiar. La formación del autoconcepto se puede estimular por medio de la conciencia de los atletas, y la planificación de la carrera profesional, y debe enfrentarse con una actitud optimista y positiva.

Evaluar de manera integral las ventajas y los dilemas de los atletas en su desarrollo profesional en función de sus condiciones físicas y haga predicciones. Combinando la estructura familiar y las condiciones económicas de los atletas con el estado de las relaciones sociales, considerando los roles desempeñados en cada etapa de su desarrollo de carrera profesional actual y futura, proponen planes de desarrollo y transformación de carrera profesional racionalizados.
Exploración: Explora una orientación profesional que se adapte a tus propias características.
Usar herramientas científicas, objetivas y precisas de evaluación y seguimiento de la carrera profesional para lograr la transformación del empleo; proporcionando una capacidad de apoyo para las características ocupacionales, las características de la demanda y el estado mental de acuerdo con las metas y necesidades futuras de los atletas.
Establecimiento:

Evaluación precisa y apoyo
La profesionalización de la planificación de la carrera profesional y el establecimiento de una base de datos de talentos son prioridades clave. Para el establecimiento de una plataforma profesional de consultoría deportiva, con el fin de garantizar una la futura transición y entrenamiento de los atletas.
Mantenimiento:

Establecer una plataforma de consultoría para la formación periódica.

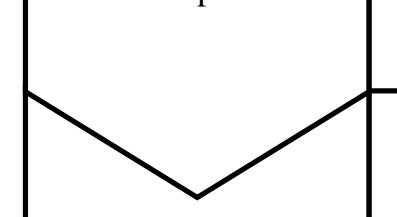

Declive: Establecer un sistema comprensivo de integración y continuar la herencia
Establecer un sistema de trabajo integral de orientación profesional deportiva para brindar apoyo, administración, evaluación y mediación para las etapas profesionales de los atletas, y brindar una protección integral de múltiples aspectos como competencias, lesiones, pensamientos, psicología y capacitación vocacional. 


\section{Bibliografía}

- Agresta, M., C., Regina, M., Brandão, F., De Paula, S., C., \& Rebustini, F. (2003, July). The problem of the sport career termination. Paper presented at the 11th European Congress of Sport Psychology, Copenhagen, Denmark. Retrieved from http://www.ktn.gv.at/24078_DEPDF-Abstractband_FEPSAC.pdf. https://doi.org/10.1037/e547922012-007

- Blinde, E. M., \& Stratta, T. M. (1992). The "sport career death" of college athletes:Involuntary and unanticipated sports exits. Journal of Sport Behavior, 15, 3-20.

- Cecić Erpič, S. (2000). Konec vrhunske športne kariere in napoved prilagoditve na pos portno z ivljenje [Elite sports career transition and prediction of the adaptation to the post-sports life] (Unpublished doctoral dissertation). University of Ljubljana, Ljubljana, Slovenia.

- Conzelmann, A., \& Nagel, S. (2003). Professional careers of the German Olympic athletes. International Review for the Sociology of Sport, 38, 259-280. https://doi.org/10.1177/10126902030383001

- Fraser, L., Fogarty, G., \& Albion, M. (2010). A longitudinal analysis of the transition to retirement from elite sport: Athletic identity, life satisfaction, and career indecision. Journal of Science and Medicine in Sport, 12, e174. https://doi.org/10.1016/j.jsams.2009.10.366

- Huang, Z. (2002). Athletic career transition in former Chinese elite athletes: An empirical investigation and cross-cultural comparison with finding from Germany (Unpublished doctoral dissertation). München University, Bundeswehr, Germany.

- Kane, M. A. (1991). The metagonic transition: A study of career transition, marital stress and identity transformation in former professional athletes (Doctoral dissertation). Available from ProQuest Dissertations and Theses Database. (ProQuest document ID: 746446081)

- Liu, F., \& Li, Q. (2007). The Influence of Group Characteristics on Career Development after Retirement of Table Tennis Athletes in China. Journal of Beijing Sport University. doi: CNKI:SUN:BJTD.0.2007-12-043

- Muscat, A. C. (2010). Elite athletes' experience of identity changes during a career-ending injury: An interpretative description. (Unpublished doctoral dissertation). University of British Columbia, Vancouver, Canada.

- Perna, F. M., Ahlgren, R. L., \& Zaichkowsky, L. (1999). The influence of career planning, race, and athletic injury on life satisfaction among recently retired collegiate male athletes. The Sport Psychologist, 13, 144-156. Retrieved from http://hk.humankinetics.com/tsp/journalAbout.cfm https://doi.org/10.1123/tsp.13.2.144

- Stankovich, C. E. (1998). The effectiveness of a career development intervention program designed to assist student-athletes through the sport retirement transition (Doctoral dissertation). Available from ProQuest Dissertations and Theses Database. (ProQuest document ID: 737999211)

- Stråhlman, O. (2006). Elite sport retirement process: career analysis of former Swedish elite athletes. (Unpublished doctoral dissertation). Göteborg University, Göteborg, Sweden. 
- Stronach, M., \& Adair, D. (2010). Load of the square ring: Future capital and career transition issues for elite indigenous Australian boxers. Cosmopolitan Civil Societies Journal, 2, 46-70. Retrieved from http://epress.lib.uts.edu.au/journals/index.php/mcs/article/viewArticle/1512. https://doi.org/10.5130/ccs.v2i2.1512

- Wilder, K. C. (1999). The career transition process: Perspectives of elite female cyclists (Doctoral dissertation). Available from ProQuest Dissertations and Theses Database. (ProQuest document ID: 733522861)

- Zaichkowsky, L., King, E., \& McCarthy, J. (2000). The end of an era: The case of forced transition involving Boston University football. In D. Lavallee \& P. Wylleman (Eds.), Career transitions in sport: international perspectives (pp. 195-205). Morgantown, WV: Fitness Information Technology. 